\title{
Fabrication and Mechanical Properties of Carbon-fiber-reinforced Polymer Composites with Lead-free Piezoelectric Nanoparticles
}

\author{
Hiroki Kurita, Zhenjin Wang, Hiroaki Nagaoka, and Fumio Narita*
}

Department of Materials Processing, Graduate School of Engineering, Tohoku University, Sendai 980-8579, Japan

(Received February 3, 2020; accepted February 27, 2020)

Keywords: piezoelectricity, polymer-matrix composites, bending strength, energy harvesting

Wireless sensor networks (WSNs) are one of the key factors in realizing an Internet of Things (IoT) society. Piezoelectric materials that can convert mechanical energy into electric energy directly are highly promising as energy-harvesting materials for supplying power to wireless sensors. However, it is well known that lead zirconate titanate (PZT), which has the highest piezoelectric performance, is harmful to the environment. Moreover, the high density and brittleness of the piezoceramics hinder the fabrication of a long-life energy-harvesting device. In this study, we fabricated 0-3 structure piezoelectric composite materials consisting of lead-free piezoceramic nanoparticles, epoxy resin, and carbon-fiber-reinforced polymer (CFRP) and evaluated their mechanical properties. The maximum strain energy of the piezoelectric resin/CFRP composites was $20-40 \%$ larger than that of piezoelectric/epoxy composites. The maximum stress and flexural modulus of the lead-free piezoelectric potassium sodium niobate (KNN) nanoparticles/CFRP composite were approximately 5-10\% larger than those of the barium titanate (BTO) nanoparticles/CFRP composite. Consequently, it is likely that better energy harvesting performance and mechanical properties can be obtained by using KNN nanoparticles than by using BTO nanoparticles.

\section{Introduction}

The construction of wireless sensor networks (WSNs) has led to the development of the Internet of Things (IoT) society. To realize true WSNs, the method of supplying energy must be established. Batteries have long been utilized as the main power source of WSN nodes, where the usage time of the node is determined by the remaining amount of energy in the battery. Moreover, batteries must be changed or recharged, and these operations decrease the efficiency of the network and increase the cost in many applications. ${ }^{(1)}$ Several strategies have been studied to extend the lifespan of WSNs, such as switching between active and sleep modes to reduce energy consumption and introducing energy harvesting technology to supply power to the nodes. ${ }^{(2,3)}$ Energy harvesting technology in particular has attracted considerable attention as an alternative method of powering nodes in wireless communication, and ways to collect energy

*Corresponding author: e-mail: narita@material.tohoku.ac.jp

https://doi.org/10.18494/SAM.2020.2820 
from direct force input such as vibrations and movements have been studied. ${ }^{(4-7)}$ Narita and Fox summarized the recent progress in piezoelectric ceramics and polymers, magnetostrictive alloys, and magnetoelectric multiferroic composites for energy harvesting applications. ${ }^{(8)}$ The challenges presented by the materials in fabrication, characterization, modelling and simulation, and durability and reliability have also been studied.

Piezoelectric material is one of the solutions for energy harvesting, which is the direct conversion of mechanical energy to electric energy. ${ }^{(9)}$ There are several forms of piezoelectric material, such as quartz, polymers, and ceramics. The piezoelectric ceramics (piezoceramics) have been widely used as sensors and actuators. The piezoelectricity of piezoceramics generally comes from their perovskite structure. In this structure, the charge in the positional relationship with positive or negative charge causes the generation of an electric field. In addition, the piezoelectricity is characterized by the piezoelectric constant $d_{33}$. In a one-dimensional problem, the relationship between $d_{33}$ and electric displacement $D$ is given by

$$
D=d \sigma+\varepsilon E,
$$

where $\sigma$ is the stress, $E$ is the electric field intensity, and $\varepsilon$ is the dielectric constant. $d_{33}$ is the piezoelectric constant when mechanical loading and poling are in the same direction. Piezoceramics normally have high $d_{33}$. For example, lead zirconate titanate (PZT) shows $d_{33}=225-590 \mathrm{pC} / \mathrm{N}$ and barium titanate (BTO) shows $d_{33}=191 \mathrm{pC} / \mathrm{N}$, whereas polyvinylidene fluoride (PVDF) shows $d_{33}=24-37 \mathrm{pC} / \mathrm{N}^{(10,11)}$ However, there are several problems regarding the application of piezoceramics. The use of PZT with the largest $d_{33}$ is restricted because of its environmental toxicity. ${ }^{(12)}$ On the other hand, the high density and brittleness of piezoceramics result in the shortening of the device lifetime in WSNs.

To overcome these problems, two approaches have mainly been considered. The first approach is to use lead-free piezoceramics such as BTO and potassium sodium niobate $(\mathrm{KNN}) .{ }^{(13,14)}$ These piezoceramics also have a perovskite structure and show relatively large piezoelectric constants. They have widely been considered as the main alternative piezoceramics to lead-based ones. ${ }^{(15,16)}$ Another approach is to use a combination of epoxy resin and carbon-fiber-reinforced polymer (CFRP). ${ }^{(17)}$ Epoxy resin has outstanding mechanical properties, thermal stability, chemical resistance, adhesion properties, and dimensional stability arising from the highly crosslinked structures of the cured epoxy resin. ${ }^{(18)}$ CFRP is a composite material that effectively exploits the advantages of carbon fibers framed by a polymer matrix. Its superior strength-to-weight ratio and good corrosion resistance and fatigue properties accelerate its application in engineering, such as in reinforcing, strengthening, and retrofitting structures. ${ }^{(19)}$ In previous studies, Tanimoto combined CFRP and bulk PZT material to damp the vibration of CFRP beams. ${ }^{(20)}$ However, PZT and CFRP were bonded, not fabricated as a composite. Saidina et al. studied a BTO/epoxy composite thin film for capacitor application. ${ }^{(21)}$ Sundar et al. developed a PZT/epoxy composite that showed a maximum $d_{33}$ of $0.56 \mathrm{pC} / \mathrm{N}$ when the volume fraction of PZT was equal to $60 \%{ }^{.22)}$ Nevertheless, the fabrication and polarization of the piezoelectric composites combined with CFRP have not yet been studied to the best of our knowledge. 
In this study, we fabricated 0-3 structure piezoelectric composite materials consisting of leadfree piezoceramic nanoparticles, epoxy resin, and CFRP. After confirming the polarization of the piezoelectric resin composite material via electrical testing, we evaluated their mechanical properties.

\section{Experimental Procedure}

Figure 1 shows the fabrication process of the piezoelectric nanoparticle-dispersed epoxy resin composites (piezoelectric/epoxy composites). KNN nanoparticles (Nippon Chemical Industrial Co., Ltd.) and BTO nanoparticles (Nippon Chemical Industrial Co., Ltd.) were employed as piezoelectric filler. Piezoelectric nanoparticles, bisphenol-F epoxy resin (Daido Co., Ltd.), and hardener (Mitsubishi Chemical Co., Ltd.) were mixed for $10 \mathrm{~min}$ and defoamed for $10 \mathrm{~min}$. The volume fraction of piezoelectric nanoparticles was controlled to be $30 \mathrm{vol} . \%$ in the final composite.

The mixture was spread on a mold and cured at $80{ }^{\circ} \mathrm{C}$ for $180 \mathrm{~min}$. After curing, the specimen was cut and polished to the dimensions as shown in Figs. 2(a) and 3(a). The specimen was polarized using a corona poling system (ELC-01N, Element Co., Ltd.) for $30 \mathrm{~min}$ at an electric field of $16 \mathrm{kV} / \mathrm{mm}$. In addition, the poling temperatures were $75{ }^{\circ} \mathrm{C}$ for $\mathrm{KNN}$ nanoparticles/epoxy and $65{ }^{\circ} \mathrm{C}$ for BTO nanoparticles/epoxy, respectively. ${ }^{(13,14)}$ Poling was performed along the thickness direction, and $\mathrm{Au}$ electrodes were coated on the upper and lower surfaces using a sputtering machine (SC-701 Mk II, Sanyu Electron Co., Ltd.).

Electrical properties of the specimens were measured as shown in Figs. 2(b) and 3(b), and the piezoelectric constant $d_{33}$ and output power density $W$ were estimated. Output power density was calculated as

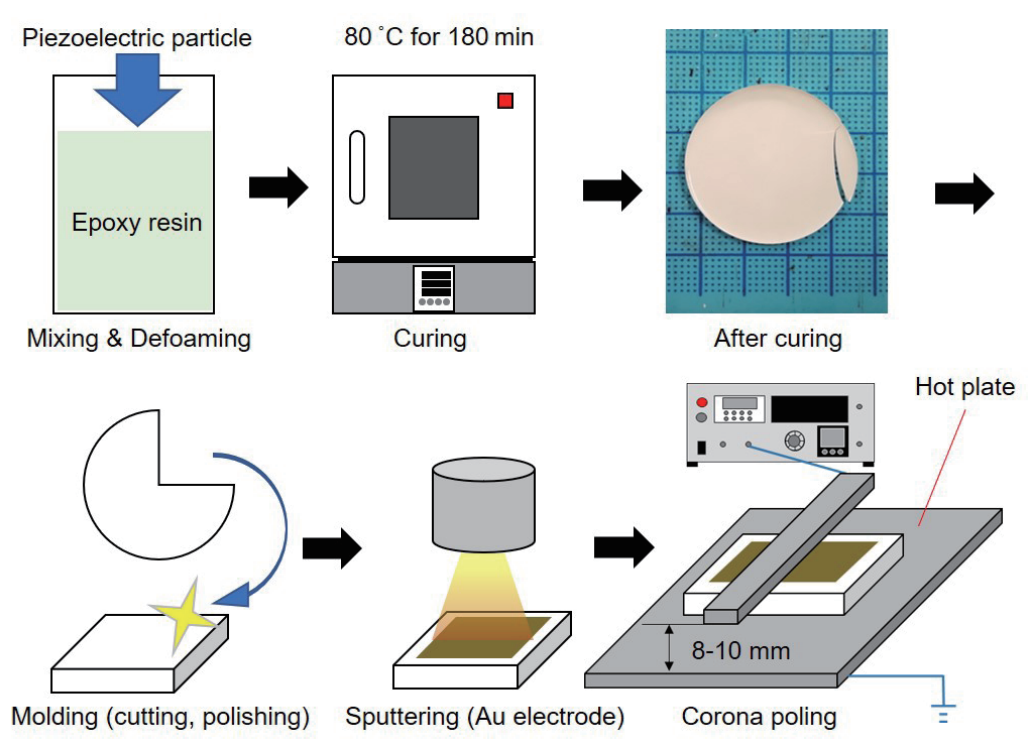

Fig. 1. (Color online) Fabrication method of piezoelectric resin specimen. 


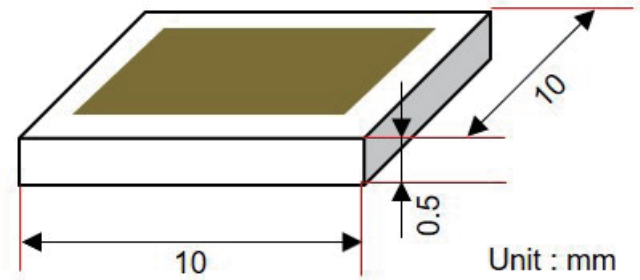

(a)

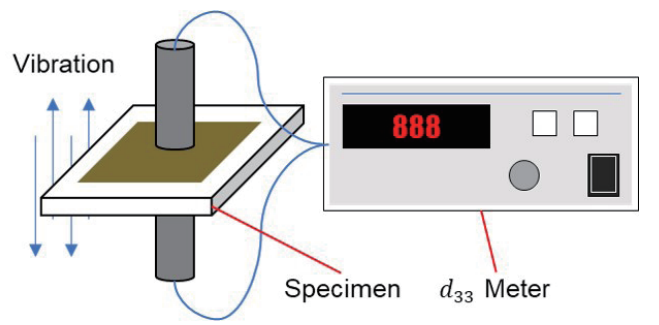

(b)

Fig. 2. (Color online) $d_{33}$ measurement: (a) piezoelectric resin specimen dimensions and (b) measurement conditions and equipment.

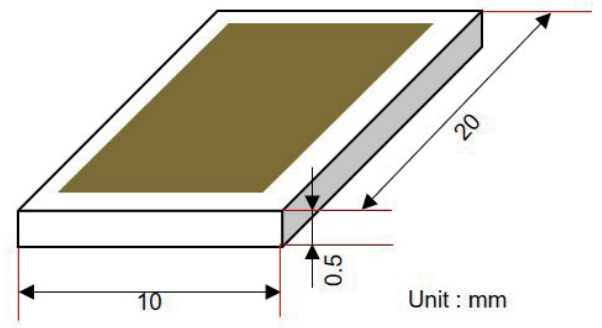

(a)

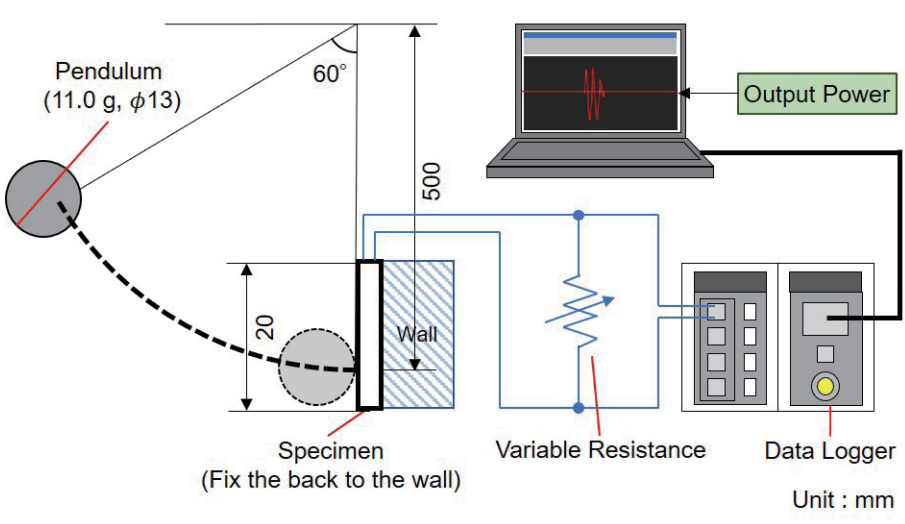

(b)

Fig. 3. (Color online) Output power measurement: (a) piezoelectric resin specimen dimensions and (b) measurement conditions and equipment.

$$
W=\frac{V_{o u t}^{2}}{R \cdot V},
$$

where $V_{\text {out }}$ is the output voltage, $R$ is the load resistance, and $V$ is the volume of the specimen.

Figure 4 shows the fabrication process of CFRP composites with piezoelectric resin (piezoelectric resin/CFRP composites). The piezoelectric nanoparticles/epoxy composites were sandwiched by glass-fiber-reinforced polymer (GFRP) and CFRP laminates. CFRP laminate was prepared by laminating CFRP prepregs in the cross-ply $\left(0 \% 90^{\circ} / 0^{\circ} / 90^{\circ}\right)$ format. After lamination, the specimen was cured at $125^{\circ} \mathrm{C}$ for $45 \mathrm{~min}$ in an oven.

The modified small punch (MSP) test ${ }^{(23)}$ and the three-point bending test were carried out. Figure 5(a) shows the cross section of the piezoelectric resin/CFRP composite and the piezoelectric nanoparticles/epoxy composite for comparison. The specimens had the dimensions of $10 \times 10 \times 1.5 \mathrm{~mm}^{3}$. Figure 5(b) shows the punch and specimen holder designed for MSP tests. The specimen holder consisted of an upper and a lower die. A universal testing machine (Autograph AG-50kNXD, Shimadzu Corporation) with a $5 \mathrm{kN}$ load cell was used 


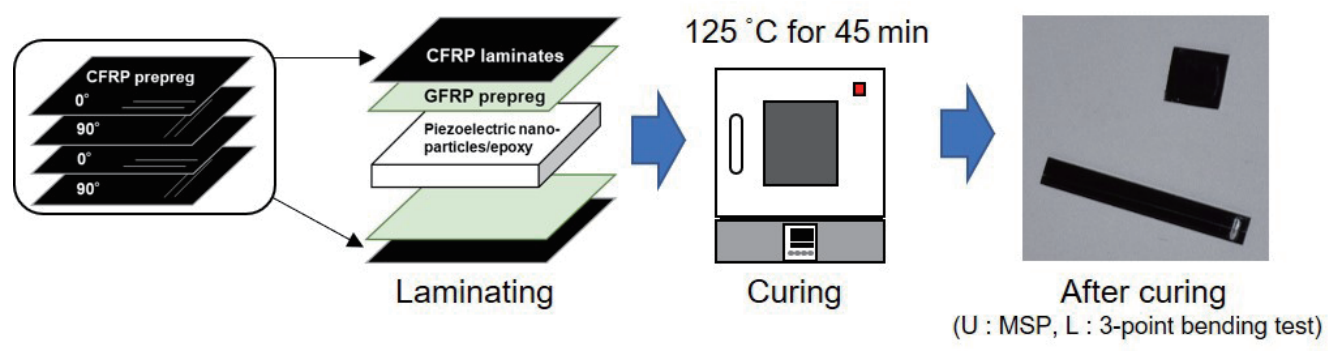

Fig. 4. (Color online) Dimensions and fabrication of piezoelectric resin/CFRP composite specimen.

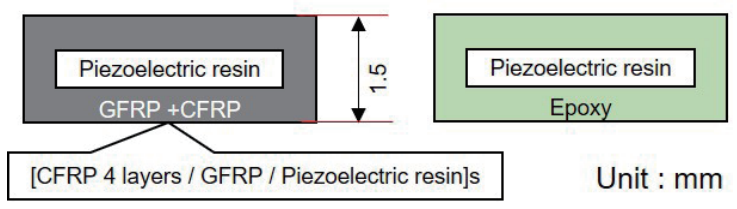

(a)

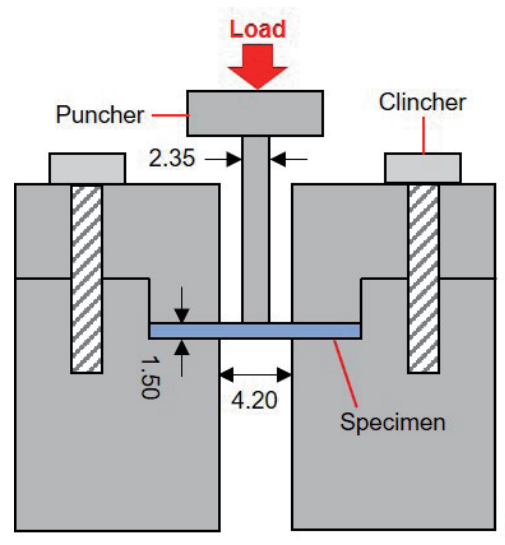

(b)

Fig. 5. (Color online) Schematic of MSP: (a) piezoelectric resin/CFRP composite and (b) test conditions.

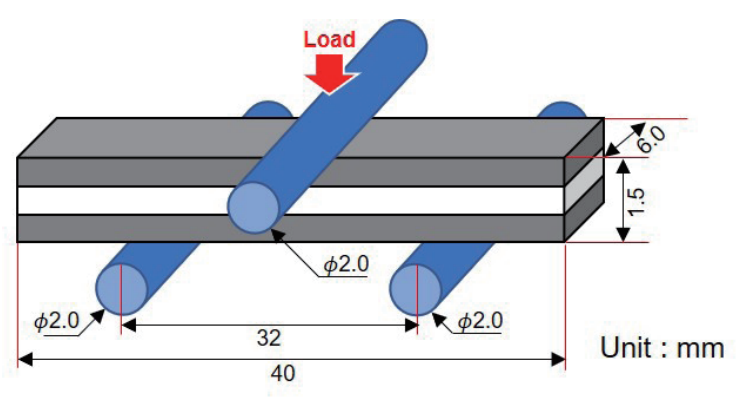

(a)

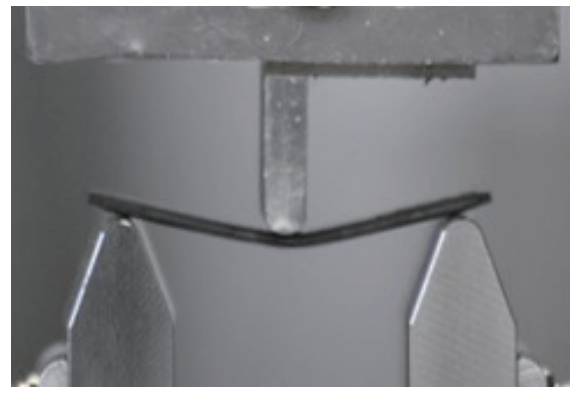

(b)

Fig. 6. (Color online) Three-point bending test: (a) schematic of test and (b) equipment and specimen.

for the MSP test with the machine crosshead speed of $0.1 \mathrm{~mm} / \mathrm{min}$. The strain energies of piezoelectric resin/CFRP composite and piezoelectric nanoparticles/epoxy composite were compared to clarify the mechanical reinforcement resulting from the combination with CFRP.

The three-point bending test was performed to investigate the bending properties and fracture process of piezoelectric resin/CFRP composite. The specimens were cut to $40 \times 6.0 \times 1.5 \mathrm{~mm}^{3}$ as shown in Fig. 6(a). The loading nose and support roller diameters were 
$2.0 \mathrm{~mm}$. The support span length was chosen to be $32 \mathrm{~mm}$. The three-point bending test was carried out with reference to JIS K 7017. The test condition was the $2 / 5$ size of JIS K 7017 except for the thickness of the specimen (see Fig. 6). A universal testing machine (Autograph AG-50kNXD, Shimadzu Corporation) was used for the three-point bending test with a machine crosshead speed of $0.1 \mathrm{~mm} / \mathrm{min}$.

\section{Results and Discussion}

Table 1 shows the density of the piezoelectric nanoparticles/epoxy specimen. The densities of KNN nanoparticles/epoxy and BTO nanoparticles/epoxy composites were 2.34 and $2.69 \mathrm{~g} / \mathrm{cm}^{3}$, respectively. These densities were approximately half that of the bulk material, ${ }^{(24,25)}$ confirming weight saving as a result of combining piezoelectric nanoparticles and epoxy.

The piezoelectric constant $d_{33}$ was measured and the results are shown in Table 2. The $d_{33}$ values of KNN nanoparticles/epoxy and BTO nanoparticles/epoxy composites were 13.6 and $12.3 \mathrm{C} / \mathrm{N}$, respectively. Table 2 also shows the $d_{33}$ values for the bulk of each piezoelectric material. ${ }^{(10,20)}$ Because the piezoelectric nanoparticles/epoxy composite has the 0-3 structure (in this case, the piezoelectric particle is the 0-dimensional filler and epoxy is the three-dimensional matrix), the piezoelectric properties were decreased compared with those of the bulk.

The output power density against variable resistance $(1.0-10 \mathrm{k} \Omega$ ) is shown in Fig. 7. The optimum resistance was approximately $5 \mathrm{k} \Omega$, and the output power densities of KNN nanoparticles/epoxy and BTO nanoparticles/epoxy composites were approximately 0.606 and $0.371 \mu \mathrm{W} / \mathrm{cm}^{3}$, respectively.

Table 1

Density of piezoelectric nanoparticles/epoxy composites and bulk.

\begin{tabular}{lcc}
\hline \multirow{2}{*}{ Piezoelectric material } & \multicolumn{2}{c}{ Density $\left(\mathrm{g} / \mathrm{cm}^{3}\right)$} \\
\cline { 2 - 3 } & Composites & Bulk \\
\hline KNN & 2.34 & 4.51 \\
BTO & 2.69 & 6.02 \\
\hline
\end{tabular}

Table 2

Piezoelectric constant of piezoelectric nanoparticles/ epoxy composites and bulk.

\begin{tabular}{lcc}
\hline \multirow{2}{*}{ Piezoelectric material } & \multicolumn{2}{c}{$d_{33}(\mathrm{pC} / \mathrm{N})$} \\
\cline { 2 - 3 } & Composites & Bulk \\
\hline KNN & 13.6 & 127 \\
BTO & 12.3 & 191 \\
\hline
\end{tabular}

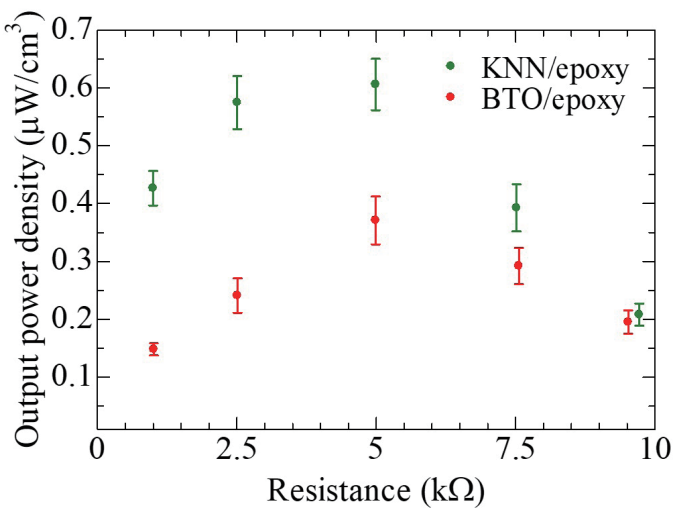

Fig. 7. (Color online) Output power density vs resistance for the piezoelectric resin composite. 
Furthermore, the mechanical properties of piezoelectric/CFRP composites were also evaluated. The load-displacement curves obtained by the MSP tests are shown in Fig. 8. According to the curve, the piezoelectric resin/CFRP specimen displayed a temporary reduction in the load gradient when the displacement was approximately $0.2 \mathrm{~mm}$. This decrease in the gradient was caused by the FRP layer. To elucidate the failure mechanism, the piezoelectric resin/CFRP composite was prepared again and its cross section was observed under loads of 1000, 1200 (before failure), and $1200 \mathrm{~N}$ (after the failure). The cross section of the specimens is shown in Fig. 9. At the load of $1000 \mathrm{~N}$, an interlaminar crack occurred at the interface

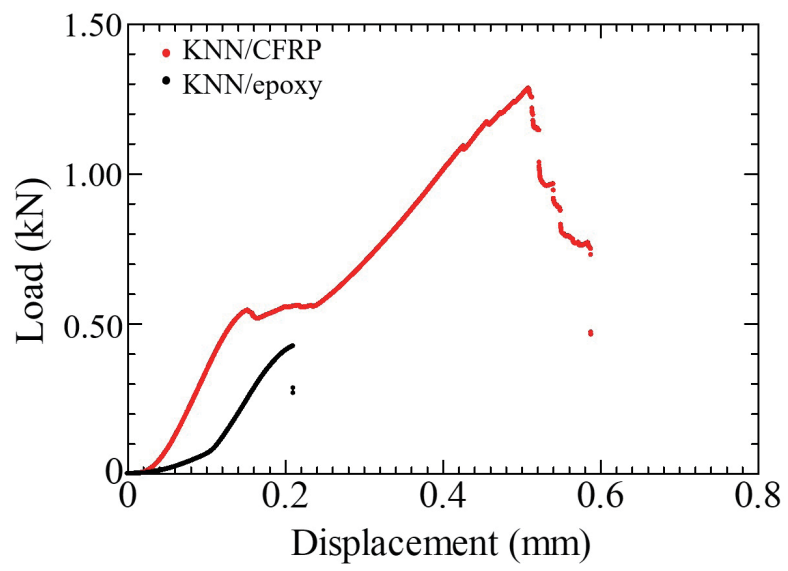

(a)

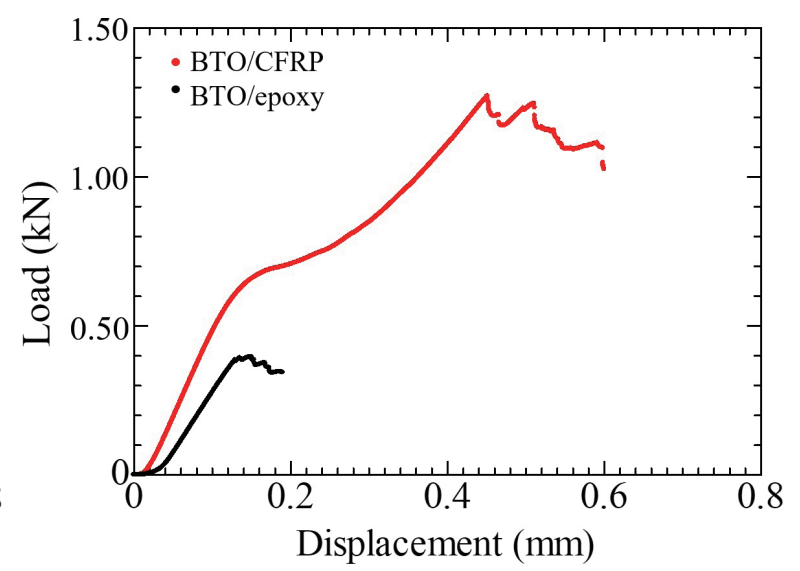

(b)

Fig. 8. (Color online) Load-displacement curve: (a) KNN nanoparticles/CFRP and KNN nanoparticles/epoxy composites and (b) BTO nanoparticles/CFRP and BTO nanoparticles/epoxy composites.

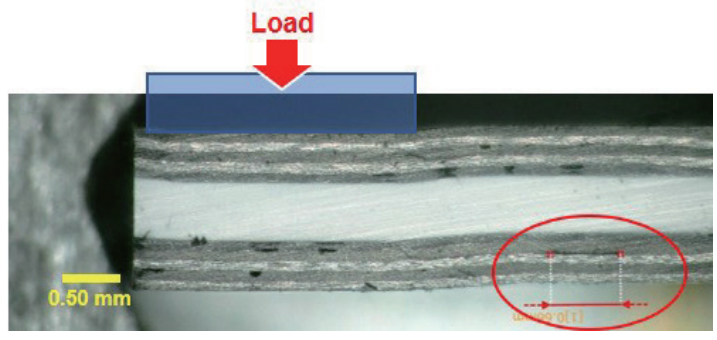

(a)

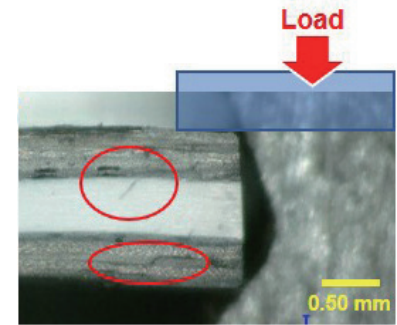

(b)

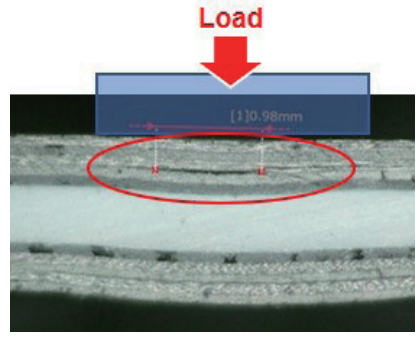

(c)

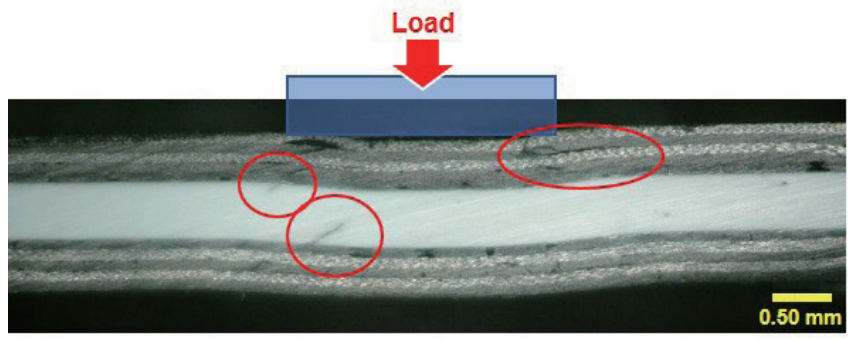

(d)

Fig. 9. (Color online) Cross section with loads of (a) $1000 \mathrm{~N}$, (b,c) $1200 \mathrm{~N}$ before failure, and (d) $1200 \mathrm{~N}$ after failure. 
between the CFRP layers. At the load of $1200 \mathrm{~N}$ (before failure), small cracks were found in the piezoelectric layer. Obviously, the two kinds of cracks extended after failure [see Fig. 9(d)].

Overall, the piezoelectric resin/CFRP composite can be utilized with the displacement smaller than $0.2 \mathrm{~mm}$. Therefore, to prove the strengthening by CFRP, strain energy was calculated by the integration of load from the displacement of 0 to $0.2 \mathrm{~mm}$. An example of the integrated area is presented in Fig. 10. Figure 11 gives the calculated maximum strain energy of the composites. According to the results, the strain energy increased about $20-40 \%$ when CFRP was incorporated, indicating the enhancement of the strength of the composite by CFRP.

The stress-strain curve obtained by the three-point bending test and the bending properties are shown in Fig. 12 and Table 3, respectively. The maximum stress $\sigma_{f}$ values of KNN nanoparticles/CFRP and BTO nanoparticles/CFRP composites were approximately 854 and

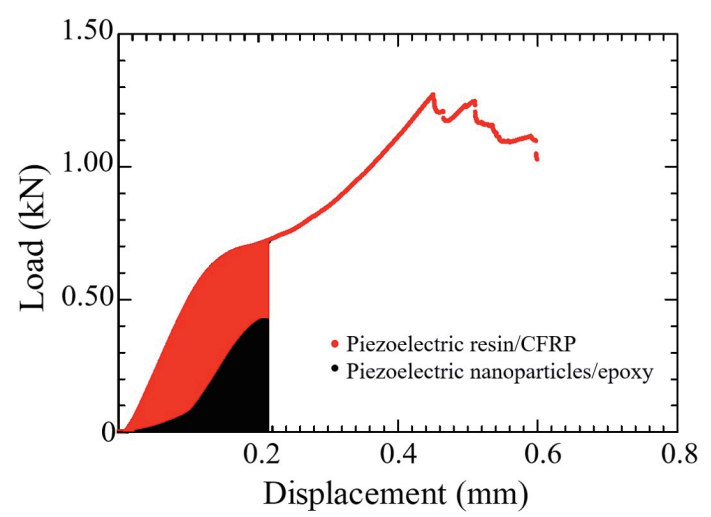

Fig. 10. (Color online) Region used to calculate strain energy.

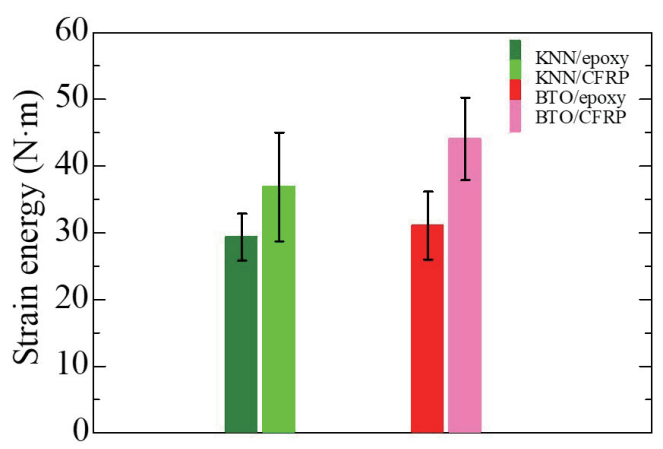

Fig. 11. (Color online) Strain energies up to the displacement of $0.2 \mathrm{~mm}$.

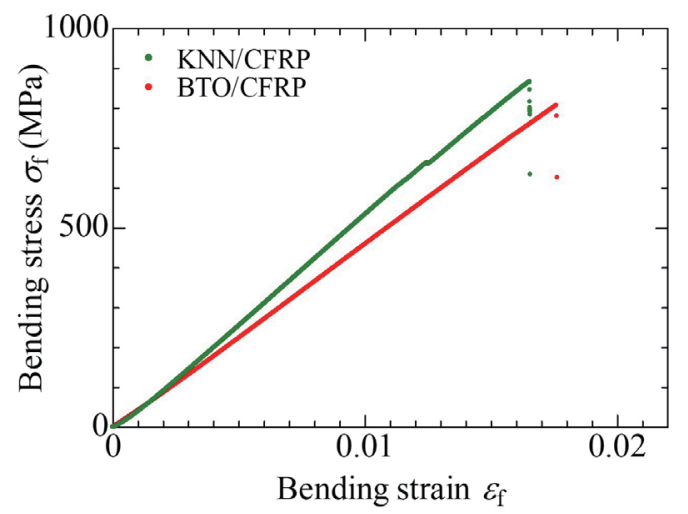

Fig. 12. (Color online) Bending stress-strain curve of CFRP composites.

Table 3

Bending properties of piezoelectric resin/CFRP.

\begin{tabular}{lcc}
\hline \multirow{2}{*}{ Measured physical properties } & \multicolumn{2}{c}{ Piezoelectric material } \\
\cline { 2 - 3 } & KNN & BTO \\
\hline Maximum stress $\sigma_{f}(\mathrm{MPa})$ & 850 & 804 \\
Maximum strain $\varepsilon_{f}(\%)$ & 1.69 & 1.87 \\
Flexural modulus $(\mathrm{GPa})$ & 46.7 & 41.6 \\
\hline
\end{tabular}




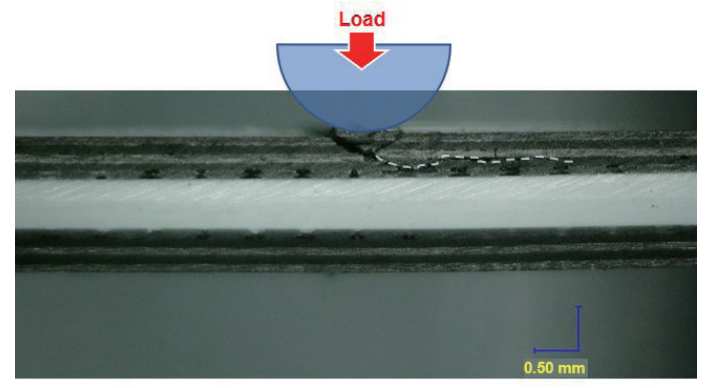

(a)

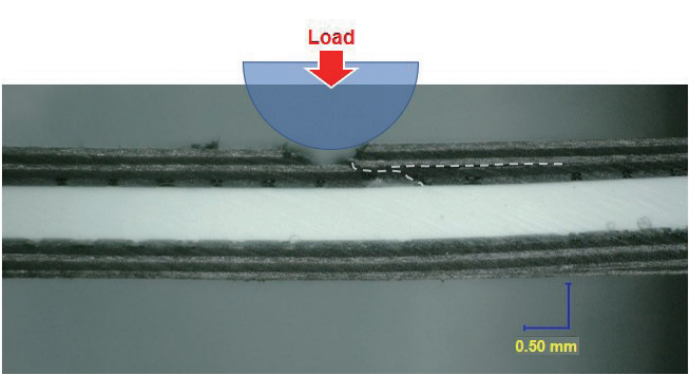

(b)

Fig. 13. (Color online) Cross-sections of (a) KNN nanoparticles/CFRP composite and (b) BTO nanoparticles/CFRP composite.

$804 \mathrm{MPa}$, respectively. In addition, the flexural moduli of KNN nanoparticles/CFRP and BTO nanoparticles/CFRP composites were approximately 46.7 and $41.6 \mathrm{GPa}$, respectively. There was an approximately $10 \%$ difference between using KNN and BTO piezoelectric material. The cross section was observed and the failure process of the piezoelectric/CFRP composite was discussed. The images observed are shown in Fig. 13. They show that CFRP first breaks on the compression side, and then the cracks propagate along the interface of CFRP for the KNN nanoparticles/epoxy composite. For the BTO nanoparticles/CFRP composite, the cracks extend from the interface to the piezoelectric layer. We believe that this is because higher compressive stress is generated in the CFRP layer owing to the lower flexural modulus of BTO nanoparticles/epoxy composite (see Fig. 12).

\section{Conclusion}

We fabricated CFRPs with lead-free piezoelectric nanoparticles and evaluated the mechanical properties of these piezoelectric resin/CFRPs by performing MSP and three-point bending tests. The maximum strain energy of the piezoelectric resin/CFRP composites was approximately $20-40 \%$ larger than that of piezoelectric nanoparticle/epoxy composites. On the other hand, the maximum stress and flexural modulus of the KNN nanoparticles/CFRP composite were approximately $5-10 \%$ larger than those of the BTO nanoparticles/CFRP composite. It seems that KNN nanoparticles contribute to the higher piezoelectric and mechanical properties to CFRPs compared with BTO nanoparticles.

\section{Acknowledgments}

The authors greatly acknowledge the support of this work by the Japan Society for the Promotion of Science (JSPS), KAKENHI under Grant No. 19H00733 and the Japan Science and Technology Agency (JST), Matching Planner Program. They would also like to thank Nippon Chemical Industrial Co. Ltd. for providing piezoelectric nanoparticles. 


\section{References}

1 S. Chamanian, H. Uluşana, Ö. Zorluc, S. Baghaeed, E. Uysal-Biyikoglu, and H. Külah: Sens. Actuators, A 249 (2016) 77.

2 A. Kozłowski and J. Sosnowski: Wireless Pers. Commun. 107 (2019) 1951.

3 Y. J. Kim, H. M. Gu, C. S. Kim, H. Choi, G. Lee, S. Kim, K. K. Yi, S. G. Lee, and B. J. Cho: Energy 162 (2018) 526.

4 Z. Yang, Y. Tan, and J. Zu: Int. J. Mech. Sci. 126 (2017) 235.

5 C. Wang, S. Wang, Q. J. Li, X. Wang, Z. Gao, and L. Zhang: Energy Convers. Manage. 163 (2018) 196.

6 J. He, T. Wen, S. Qian, Z. Zhang, Z. Tian, J. Zhu, J. Mu, X. Hou, W. Geng, J. Cho, J. Han, X. Chou, and C. Xue: Nano Energy 43 (2018) 326.

7 M-S. Lee, C-I. Kim, W-I. Park, J-H. Cho, J-H. Paik, and Y. H. Jeong: Energy 179 (2019) 373.

8 F. Narita and M. Fox: Adv. Eng. Mater. 20 (2018) 1700743.

9 Y. Shindo and F. Narita: Int. J. Mech. Mater. Design 10 (2014) 305.

10 T. R. Shrout and S. J. Zhang: J. Electroceramics 19 (2007) 113.

11 C. Wan and C. R. Bowen: J. Mater. Chem. A 5 (2017) 3091.

12 A. T. Duarte, M. B. Dessuy, M. M. Silva, M. G. R. Vale, and B. Welz: Microchem. J. 96 (2010) 102.

13 Z. Wang and F. Narita: Adv. Eng. Mater. 21 (2019) 1900169.

14 Z. Wang and F. Narita: J. Appl. Phys. 126 (2019) 224501.

15 T. Karaki, K. Yan, T. Miyamoto, and M. Adachi: Jpn. J. Appl. Phys. 46 (2007) L97.

16 J. Kim, J-H. Ji, D-J. Shin, and J.-H. Koh: Ceram. Int. 44 (2018) 22219.

17 F. Narita, H. Nagaoka, and Z. Wang: Mater. Lett. 236 (2019) 487.

18 M. Jiang, Y. Liu, C. Cheng, J. Zhou, B. Liu, M, Yu, and H. Zhang: Polym. Test. 69 (2018) 302.

19 P. Nguyen, X. Vu, and E. Ferrier: Fire Saf. J. 100 (2018) 103.

20 T. Tanimoto: Composites Sci. Technol. 67 (2007) 213.

21 D. S. Saidina, M. Mariatti, and M. J. Julie: J. Mater. Sci. 25 (2014) 4923.

22 U. Sundar, S. Banerjee, and K. Cook-Chennault: Academic J. Polymer Sci. 1 (2018) 96.

23 Y. Shindo, F. Narita, K. Horiguchi, Y. Magara, and M. Yoshida: Acta Mater. 51 (2003) 4773.

24 D. Jenko, A. Bencan, B. Malic, C. Holc, and M. Kosec: Microsc. Microanal. 11 (2005) 572.

25 A. C. Dent, C. R. Bowen, R. Stevens, M. G. Cain, and M. Stewart: J. Eur. Ceram. Soc. 27 (2007) 3739. 